\title{
Niveaux de référence diagnostiques : spécificités de la démarche française en radiologie
}

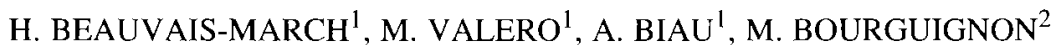

(Manuscrit reçu le 1/ octobre 2002, accepté le 26 février 2003)

RÉSUMÉ Le concept de niveau de référence diagnostique (NRD) introduit dans la CIPR 73 *Protection et sûreté radiologique en médecine » est spécifique aux expositions médicales. Dans ce domaine, en effet, l'un des trois principes de la radioprotection, la limitation réglementaire des doses, ne peut s'appliquer comme pour les expositions professionnelles et publiques. Les niveaux de référence ne sont ni des « limites de dose », ni des « doses optimales », ce sont des outils pour l'optimisation. Ils sont établis pour des examens standardisés et des patients types, et ne devraient pas être dépassés sans justification, pour des procédures courantes. Les NRD sont des indicateurs dosimétriques de la qualité des pratiques destinés à identifier les situations nécessitant une action corrective. Dans le cadre de la transposition de la directive 97/43 Euratom, le directeur général de la santé a confié à l'OPRI ${ }^{3}$, en 1999, une mission spécifique pour la mise en euvre des niveaux de référence, en concertation avec les sociétés savantes et professionnelles concernées. La méthode recommandée par la Commission européenne, dite du $75^{\mathrm{e}}$ percentile, basée sur le traitement statistique d'enquêtes dosimétriques représentatives, est à l'heure actuelle inopérante en France en raison de l'insuffisance des données. Une démarche spécifique a donc été définie. Dans un premier temps, un travail de standardisation des procédures radiologiques avec adoption des niveaux de référence européens comme point de départ a été effectué. Dans un second temps, une campagne nationale de mesures de doses co-pilotée par l'OPRI (actuellement l'IRSN et la DGSNR) ${ }^{3}$, la $\mathrm{SFR}^{3}$ et la $\mathrm{SFPM}^{3}$ est en cours de réalisation pour établir des NRD français.

ABSTRACT Diagnostic reference levels for diagnostic radiology: French implementation methods.

ICRP publication 73 (Radiological Protection and Safety in Medicine) introduced the concept of "diagnostic reference levels" (DRL), for medical exposures specifically. Because dose limits should not be applied for diagnostic or therapeutic irradiation, the approach to radiological protection is slightly different from that in occupational or public exposures. Reference levels, that are neither optimum levels, nor dose limits, are a tool for optimisation. DRL are set for standard procedures, for groups of patients or standard phantom and not for special exposures and individual

\footnotetext{
I IRSN, Service de la surveillance de l'cxposition médicale et professionnclle (SEMP), B.P. 35, 78116 Le Vésinet Cedex, France.

${ }^{2}$ Direction générale de la Sûreté nucléaire et de la radioprotection, 75572 Paris Cedex 12, France.
}

\footnotetext{
Office de protection contre les rayonnements ionisants (OPRI), Société française de radiologie (SFR), Société française de physique médicale (SFPM), Institut de radioprotection et de sûreté nucléaire (IRSN), Direction générale de la Sûreté nucléaire et la radioprotection (DGSNR).
} 
patients. They act as a test for identifying situations where patient doses are becoming unusually high and local review of procedures and equipment is required. Within the framework of implementation of Directive 97/43, the Health Directorate entrusted its radiation protection agency (past $O P R I^{3}$, at present time $D G S N R^{3}$ and $I R S N^{3}$ ) to determine the national reference dose levels in diagnostic radiology in close cooperation with relevant scientific and professional societies. The pragmatic way, recommended by European Commission, to assess DRL values is to use the third quartile values observed in wide scale surveys of typical doses for common procedures. In the absence of recent and wide-ranging data for France, in a first step, European DRI, have been adopted as starting points. To go further, a campaign of patient dose measurements has been launched by a steering committee $\left(\mathrm{OPRI}^{3}, \mathrm{SFR}^{3}\right.$ and $S F P M^{3}$ ) to allow the actual establishment of national reference values and to prepare their periodic review in a continuous process of optimization.

\section{Introduction}

De nombreux changements sont en cours dans la radioprotection française tant sur le plan de la réglementation avec la transposition des directives européennes (Conseil des communautés européennes, 1996, 1997), que sur celui de l'organisation et des structures, avec la création de la DGSNR et de l'IRSN (JO, 2002). Les évolutions concernent tous les aspects: surveillance des travailleurs, de la population et de l'environnement. Mais un domaine est plus particulièrement confronté à de réelles nouveautés: celui des expositions médicales à visée diagnostique ou thérapeutique. En effet, la radioprotection des patients fait désormais partie des obligations légales depuis l'ordonnance 2001-270 (JO, 2001a) qui a transposé en droit national la directive 97/43 Euratom. Les décrets et arrêtés d'application sont publiés ou en cours d'élaboration (JO, 2001b).

C'est dans ce contexte qu'apparaît pour la première fois en France la notion de niveau de référence diagnostique (NRD) et que se situe la mission confiée en 1999, par le directeur général de la santé à l'Office de protection contre les rayonnements ionisants (OPRI). La continuité de cette mission est aujourd'hui assurée conjointement par l'IRSN et la DGSNR. Ses objectifs sont les suivants :

- élaborer, en collaboration avec les sociétés savantes et professionnelles, les procédures écrites prévues à l'article 6 de la directive en y associant des critères de prescription et des méthodes d'optimisation des doses d'irradiation, dans les domaines de la radiologie et de la médecine nucléaire ;

- déterminer des niveaux de référence diagnostiques pour les pratiques radiologiques les plus courantes et/ou les plus irradiantes.

Le premier point a fait l'objet d'un travail spécifique, piloté avec la Société française de radiologie (SFR) et l'Association française du personnel paramédical d'électroradiologie (AFPPE), qui a abouti à la publication d'un rapport, « Les procédures radiologiques : critères de qualité et optimisation des doses » (OPRI, 2001) disponible sur les sites Internet de la SFR et de l'IRSN. 
En ce qui concerne la détermination et l'utilisation des NRD, les travaux sont en cours, mais cela pose des problèmes méthodologiques et pratiques. En effet, les études disponibles sur les doses délivrées aux patients sont soit partielles soit anciennes (Maccia et al., 1988 ; SFPH, 1988) et par ailleurs, les médecins et personnels paramédicaux pratiquant des actes irradiants sont à l'heure actuelle encore peu mobilisés sur les questions de radioprotection. Il convient donc d'adopter une démarche spécifique qui réponde aux obligations réglementaires tout en tenant compte des lacunes et des retards à combler.

\section{Définition : les niveaux de référence ne sont ni des limites réglementaires, ni des valeurs optimales}

La Commission internationale de protection radiologique (CIPR) introduit pour la première fois en 1996, dans sa publication 73, «Protection et sûreté radiologique en médecine » (ICRP, 1996), la notion de niveau de référence diagnostique. Face aux diversités d'interprétation et de mise en ceuvre de ces niveaux selon les pays et les secteurs d'activité (radiologie, médecine nucléaire...) le comité 3 de la CIPR, a décidé de revenir sur cette question en 2000 et propose un nouveau document (ICRP, 2001), en cours de discussion, destiné à compléter les recommandations initiales de la publication 73 .

Au niveau européen, un important travail de clarification a été effectué qui donne une grande cohérence au texte de la directive 97/43 (Conseil des communautés européennes, 1997). L'article 4 relatif à l'optimisation demande que « les états membres favorisent l'élaboration et l'utilisation de niveaux de référence diagnostiques ». Ces derniers sont définis dans l'article 2 comme « des niveaux de dose dans les pratiques radiodiagnostiques ou, dans le cas de produits radiopharmaceutiques des niveaux d'activité, pour des examens types sur des groupes de patients types ou sur des fantômes types, pour des catégories larges de types d'installations. Ces niveaux ne devraient pas être dépassés pour les procédures courantes si des pratiques bonnes et normales en matière de diagnostic et de performance techniques sont appliquées ». Les NRD doivent être prioritairement établis pour les examens les plus fréquents (y compris les examens de dépistage) et les plus irradiants (scanographie et radiologie interventionnelle).

Il ne s'agit donc pas de limites, au sens réglementaire du terme, et cela ne concerne pas les expositions individuelles, cas par cas. Une limitation impérative des doses, ne peut d'ailleurs se concevoir en radiologie : en effet, les doses sont délivrées volontairement pour un bénéfice attendu et sont donc dépendantes de l'objectif médical. Assujettir les doses à des limites réglementaires serait un contresens qui pourrait, dans certains cas, avoir des conséquences préjudiciables pour les patients. 
La réduction des doses au niveau strictement nécessaire, repose donc, en médecine, sur l'application des seuls principes de justification et d'optimisation avec une rigueur accrue et des outils appropriés.

Les niveaux de référence diagnostiques font partie de ces outils. Ils concernent des examens standardisés et non des procédures particulières. Ils se réfèrent à des patients «type » $(70 \pm 3 \mathrm{~kg}$ de masse corporelle, $20 \pm 2 \mathrm{~cm}$ d'épaisseur au centre du thorax) et/ou des fantômes de référence et non à des individus cas par cas.

Il faut considérer les NRD comme des indicateurs dosimétriques servant à optimiser les pratiques médicales irradiantes. Leur utilisation permet de connaître, du point de vue des doses délivrées, la qualité des équipements et des procédures, et d'engager, en cas de dépassement injustifié, des actions de contrôle et de correction.

Il doit être clair cependant que le respect des niveaux de référence n'est pas le seul critère de bonne pratique. D'une part le principe de justification des actes doit au préalable être respecté, d'autre part, délivrer des doses inférieures aux NRD n'a aucun sens si les images obtenues sont de mauvaise qualité et ne fournissent pas l'information médicale recherchée. En ce sens, la notion de niveau de référence est indissociable de celle de «la qualité informative des images ».

Enfin, il va de soi que le respect des niveaux de référence ne dispense pas de poursuivre la démarche d'optimisation des doses. Les NRD ne sont en effet pas des doses optimales. Celles-ci doivent être recherchées pour chaque procédure, en fonction des possibilités offertes par le matériel dont on dispose, des caractéristiques propres à chaque patient et de l'objectif diagnostique ou thérapeutique poursuivi.

\section{Détermination des niveaux de référence}

\subsection{Niveaux de référence diagnostiques}

Les niveaux de référence diagnostiques sont déterminés à partir d'études dosimétriques, par des experts représentant des organismes médicaux et scientifiques (Commission européenne, 1999). En France, ils sont proposés au Ministre chargé de la Santé qui les publie par arrêté (JO, 2003).

\subsection{Le choix des grandeurs dosimétriques}

La première étape en radiologie $\mathrm{e}^{4}$ consiste à choisir les grandeurs dosimétriques appropriées auxquelles on affectera ces niveaux de référence et à les définir sans

\footnotetext{
${ }^{4}$ Les NRD en médecine nucléaire, exprimés en terme d'activité administrée au patient (MBq), relèvent d'une autre problématique.
} 
ambiguïté. Pour être un outil efficace dans la démarche pratique d'optimisation, ces grandeurs doivent répondre à un certain nombre de critères :

- être clairement définies et faciles à déterminer par la mesure et/ou le calcul ;

- donner directement aux opérateurs, « au pupitre», une indication de l'importance de l'irradiation délivrée aux patients ;

- permettre des corrélations simples avec les paramètres techniques de l'examen ;

- être adaptées à tout type de matériel radiologique courant.

Le choix n'est pas évident. L'expression des doses délivrées aux patients en radiologie fait appel à un grand nombre de définitions. Les grandeurs utilisées : kerma dans l'air, dose absorbée en profondeur dans un milieu, dose à la surface d'entrée, n'ont pas le même intérêt selon qu'il s'agit de la radiologie classique ou de la scanographie, en raison des différences importantes dans la technologie des équipements et dans les conditions d'exposition. En outre, pour des raisons pratiques, des grandeurs complexes comme des produits « dose $\times$ surface » ou « dose $\times$ longueur » sont également utilisées.

La seule grandeur dosimétrique qui soit commune à tout type d'exposition est la dose efficace, et il serait a priori tentant de s'y référer pour exprimer les NRD. Mais cette grandeur ne répond pas aux critères recherchés : ce n'est pas une quantité physique mesurable et elle n'a pas de lien directement lisible avec les caractéristiques du rayonnement et les paramètres d'exposition. Définie comme la somme des doses équivalentes aux organes, pondérée en fonction de la nature des tissus, selon des facteurs proposés par la publication 60 de la CIPR (ICRP, 1991), son calcul est susceptible de révisions futures. Son intérêt essentiel réside dans le fait qu'elle permet de traduire une irradiation locale en terme d'exposition globale et de quantifier ainsi les risques d'effets stochastiques. C'est de plus une grandeur utile pour la communication avec les patients et le public. Mais ce n'est pas une donnée pertinente pour optimiser des procédures radiologiques. D’où le choix consensuel, effectué au plan international (ICRP, 2001 ; Commission européenne, 1996, 1997, 1999), de définir des niveaux de référence pour des grandeurs qui sont en apparence plus complexes pour les non-spécialistes, mais en réalité plus proches des phénomènes physiques et accessibles par la mesure.

\subsubsection{Les grandeurs dosimétriques de référence en radiologie classique}

Elles sont au nombre de deux (Commission européenne, 1996 ; OPRI, 2001) selon que l'on considère une exposition unique (une seule incidence), ou un examen complet (incidences multiples). Il s'agit de la dose à la surface d'entrée du patient 


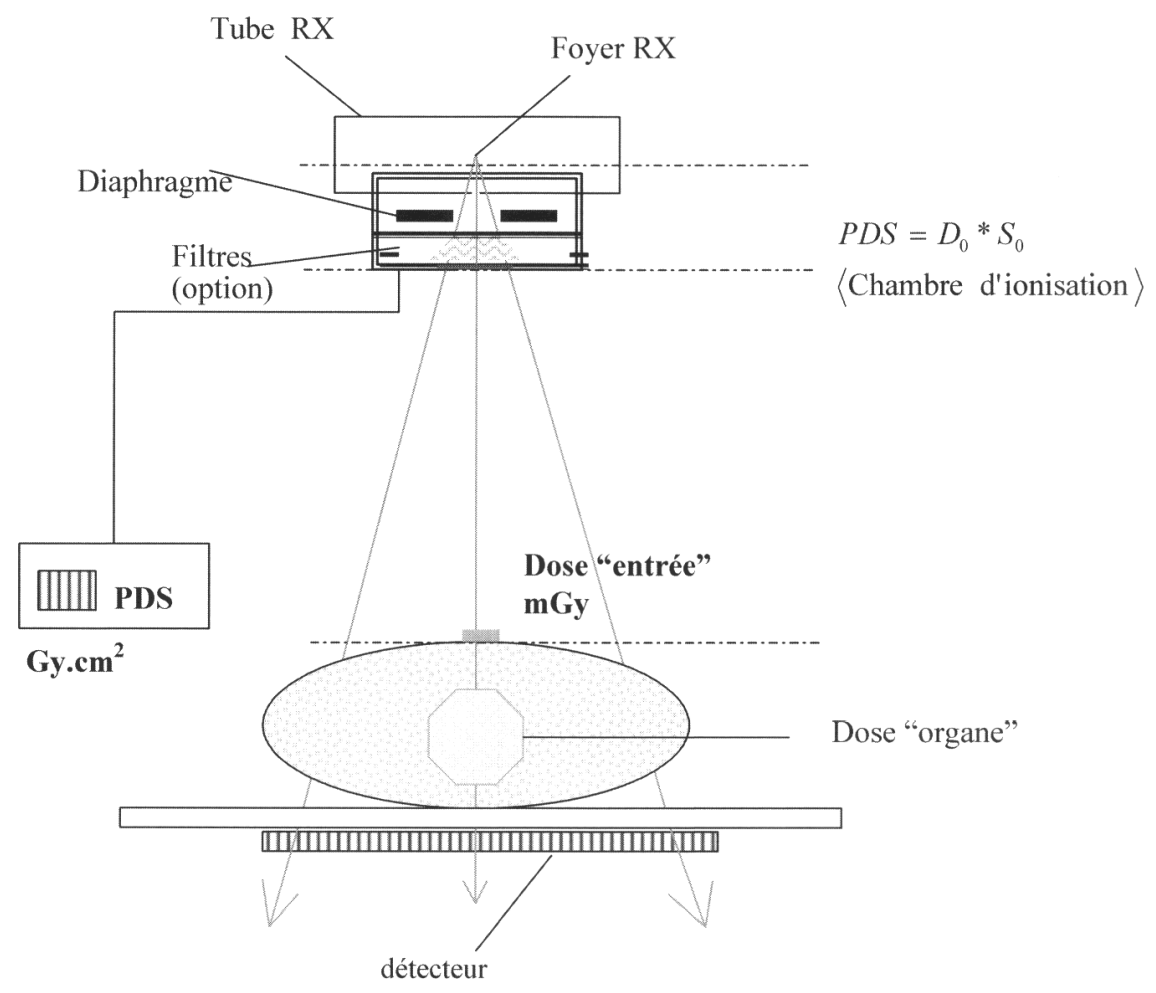

Figure 1 - Schéma d'irradiation et grandeurs dosimétriques en radiologie conventionnelle. Irradiation geometry and dose quantities in conventional radiography.

(De) exprimée en mGy et du produit dose $\times$ surface (PDS) exprimé en $\mathrm{Gy}_{\mathrm{cm}}^{2}$ (Fig. 1).

La dose à la surface d'entrée De est la dose absorbée (mGy) dans l'air au point d'intersection de l'axe du faisceau de RX et de la surface d'entrée du patient, rayonnement rétrodiffusé inclus.

Le produit (dose $\times$ surface), PDS, est le produit de la dose moyenne absorbée (Gy) dans l'air dans la section du faisceau de RX (en l'absence de milieu diffusant), par la surface de cette section $\left(\mathrm{cm}^{2}\right)$.

On utilise également ces grandeurs en radiologie interventionnelle mais la longueur et la diversité des procédures rendent le traitement des données plus complexe. 


\subsubsection{Les grandeurs dosimétriques de référence en scanographie}

La dosimétrie des scanners (Cordoliani et al., 2002 ; Nagel et al., 2000) repose sur la notion d'indice de dose de scanographie (IDS) ou CTDI (Computed Tomography Dose Index). En théorie, le CTDI est égal à l'intégrale du profil de dose $D(z)$ le long de l'axe (z) du scanner (Fig. 2), pour une rotation du tube, divisée par la valeur nominale $n T$ de la fenêtre d 'irradiation du scanner ( $n$ étant le nombre de coupes par rotation et $T$ l'épaisseur de coupe). En pratique le CTDI, exprimé en $\mathrm{mGy}$, est mesuré par chambre d'ionisation sur une longueur de $100 \mathrm{~mm}$ et sa valeur «normalisée » est rapportée à une charge de $1 \mathrm{mAs}$. Mais il existe de nombreuses variantes dans les définitions du CTDI, liées aux conditions de mesures. En particulier on peut s'intéresser au CTDI dans l'air, ou au CTDI dans un fantôme cylindrique de plexiglas de dimension standard. Dans ce dernier cas, on mesure le CTDI au centre ou en périphérie du fantôme, l'écart entre les deux valeurs étant fonction du diamètre du cylindre et des caractéristiques du faisceau de RX.

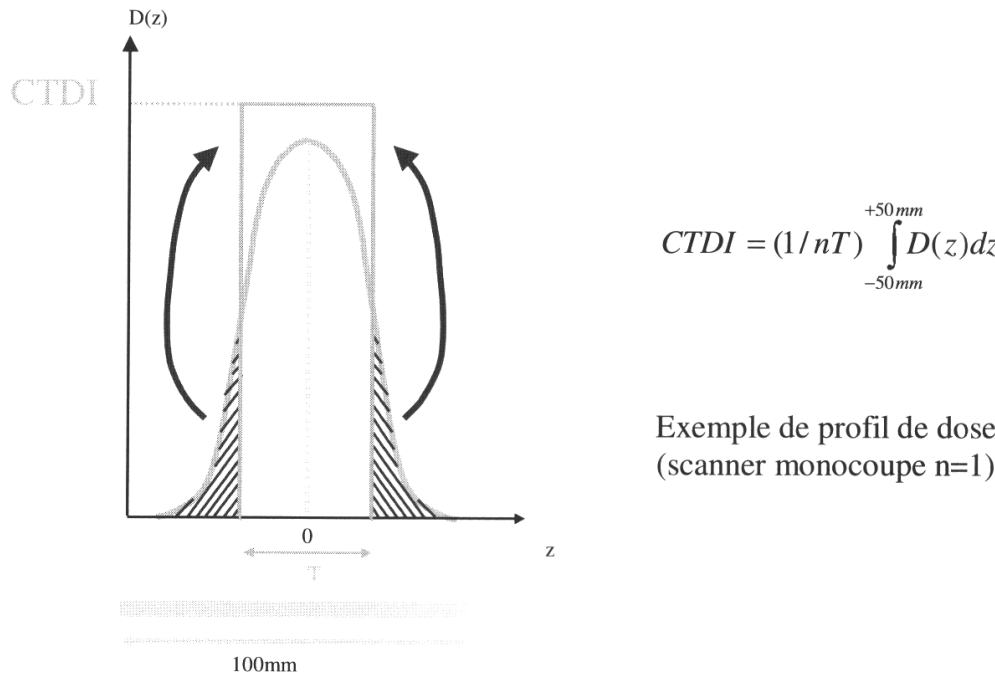

Figure 2 -Schématisation de l'IDS (ou CTDI). La zone hachurée représente la fraction de la dose ne contribuant pas à la formation de l'image, mais absorbée par le patient. Cette dose est prise en compte dans la valeur du CTDI, représentée par la surface du rectangle (égale à la surface sous la courbe de profil de dose), pour $T=10 \mathrm{~mm}$.

Illustration of the CTDI. The CTDI value is equivalent to the rectangular area (for $T=10 \mathrm{~mm}$ ), which is equal to the area under the dose profile. Hachured areas indicate dose delivered outside the imaged section. 
Les experts de la Commission européenne on choisi d'adopter deux grandeurs (Commission européenne, 1997, 1999) pour exprimer les niveaux de référence d'un examen scanographique : l'indice de dose de scanographie pondéré (IDSP) et le produit dose $\times$ longueur (PDL).

L'IDSP ou CTDIw (Weighted Computed Tomography Dose Index) est une combinaison linéaire des CTDIc et CTDIp mesurés respectivement au centre et en périphérie d'un fantôme cylindrique simulant la tête (diamètre $16 \mathrm{~cm}$ ) ou le corps (diamètre $32 \mathrm{~cm}$ ) d'un patient type, avec les paramètres d'exposition propres à l'examen considéré (tension aux bornes du tube RX, largeur de la fenêtre d'irradiation, nombre de mAs, etc.)

$$
\text { IDSP = CTDIw = (1/3 IDScentre + 2/3 IDSpériphérie }) .
$$

Le produit (dose $\times$ longueur), exprimé en $m G y \mathrm{~cm}$, s'exprime en pratique par :

$$
\mathrm{PDL}=\mathrm{CTDIw} \times \mathrm{L}
$$

L (cm) étant la longueur du volume irradié par le faisceau primaire au cours d'une acquisition: cette longueur ne doit pas être confondue avec celle du volume exploré, son calcul tient compte de l'intervalle entre les coupes en mode incrémentiel et du pas de l'hélice en mode hélicoïdal (Cordoliani et al., 2002). Le PDL de l'examen complet est égal à la somme des PDL pour chaque acquisition. L'intérêt du PDL est de quantifier non seulement la dose délivrée localement, mais également l'importance du volume irradié, ce qui permet d'exprimer de façon plus globale l'exposition du patient.

\subsection{L'adoption des valeurs numériques des niveaux de référence}

La Commission européenne recommande (Commission européenne, 1999) une méthode empirique, partant de la réalité des pratiques médicales pour aller vers une réduction progressive des doses délivrées aux patients. C'est la méthode statistique dite du $75^{\mathrm{e}}$ percentile, déjà mise en æuvre par plusieurs pays européens, dont la Grande Bretagne, depuis une vingtaine d'années (NRPB, 1999).

On adopte comme niveau de référence la valeur du $75^{\mathrm{e}}$ percentile de la distribution des doses mesurées (Fig. 3), pour une procédure donnée, sur un grand nombre de patients répartis dans un grand nombre de centres, représentatifs de la pratique radiologique d'un pays.

Le NRD n'est donc pas une moyenne (M), mais, pour chaque pratique, la valeur en-dessous de laquelle se situent 75 \% des mesures. Il s'agit d'une approche 


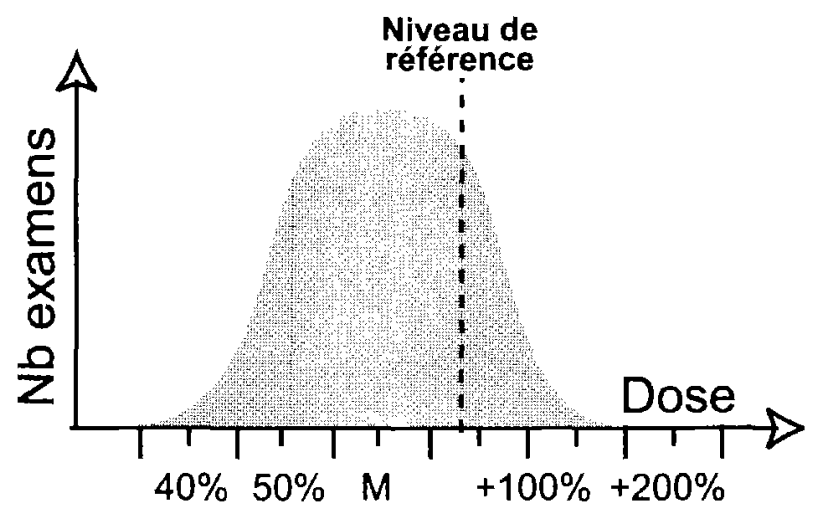

Figure 3 - Représentation schématique du $75^{e}$ percentile d'une distribution de doses (Cordioliani et al, 2002).

Schematic display of the 75 th percentile of a dose distribution.

pragmatique : ceci revient à considérer que le quart des examens correspondant aux doses les plus élevées ne sont pas réalisés dans des conditions acceptables, du point de vue de la radioprotection du patient. L'objectif est d'identifier et d'éliminer les causes de ces doses inutilement délivrées. Si cela est réellement fait dans la pratique, à l'échelle d'un pays, les enquêtes dosimétriques successives feront apparaître une évolution des courbes de distribution de doses et un abaissement de la valeur du $75^{\mathrm{e}}$ percentile (NRPB, 1999). La valeur des NRD est donc par nature évolutive, sachant qu'il y a obligatoirement une limite inférieure conditionnée par les performances de la technologie et par la nécessité d'obtenir des images d'une qualité informative satisfaisante.

La directive 97/43 Euratom recommande à chaque état membre (Commission européenne, 1999) de déterminer ses propres niveaux de référence, ou à défaut d'adopter ceux proposés par la Commission européenne (Tabs. I et II), qui découlent de la mise en œuvre de la méthode du $75^{\mathrm{c}}$ percentile, appliquée à une sélection de services de radiologie représentatifs des différents pays de la communauté.

Proposer dès à présent des valeurs de NRD spécifiques à la France serait prématuré : en effet, les mesures dont on dispose à ce jour sont insuffisantes (Maccia et al., 1988 ; SFPH, 1988), pour permettre un traitement statistique fiable au niveau national. 


\section{TABLEAU I}

NRD européens pour des examens de radiologie classique, exprimés en dose à la surface d'entrée (De) pour une exposition.

European DRL for standard examinations expressed in term of entrance surface dose per single view.

\begin{tabular}{|c|c|}
\hline Prom & 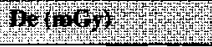 \\
\hline Thorax de face & 0.3 \\
\hline Thorax de profil & 1.5 \\
\hline Rachis lombaire de face & 10 \\
\hline Rachis tombaire de prof & 30 \\
\hline Abdomen sans préparation & 10 \\
\hline Bassinde face & 10 \\
\hline Mammographie (différentes incidences) & 10 \\
\hline Crâne de face & $\therefore 5$ \\
\hline Crâne de profil & 3 \\
\hline
\end{tabular}

\section{TABLEAU II}

NRD européens pour des examens de scanographie, exprimés en indice de dose de scanographie pondéré (IDSP ou CTDIw) et en produit dose $\times$ longueur (PDL).

European DRL for CT examinations expressed in terms of CTDIw and DLP.

\begin{tabular}{|c|c|c|}
\hline Th phent & 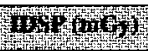 & 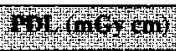 \\
\hline Scanographic du crâne standard & 58 & 1050 \\
\hline Stanggraphie du thorax standand & 27 & 650 \\
\hline Scanographic de l'abdomen standard & 33 & 770 \\
\hline Scanographle du pelvis standard & 33 & 570 \\
\hline
\end{tabular}

Le comité de pilotage de la mission OPRI-SFR (OPRI, 2001) a par conséquent décidé d'opérer en deux étapes :

- effectuer dans un premier temps, un travail de standardisation des procédures radiologiques avec adoption, après les avoir validés, des niveaux de référence européens ;

- engager dans un deuxième temps, une campagne nationale de mesures de doses en radiologie pour définir des niveaux français.

Un « état des lieux » a donc été réalisé, comportant une étude bibliographique (les données françaises existantes ont été analysées et comparées avec les données internationales) complétée par un certains nombre de mesures ayant valeurs de tests significatifs. Une étude dosimétrique a été effectuée par le service de dosimétrie de l'OPRI dans le cadre d'une mission antérieure sur l'irradiation médicale des patients, confiée en 1998 par le secrétaire d'état à la santé aux 


\section{TABLEAU III}

Doses à la surface d'entrée (en mGy), mesurées par dosimètres thermoluminescents, sur fantôme anthropomorphe type RANDO, pour 10 examens, dans 3 services hospitaliers de radiologie de la région parisienne en 1999 (OPRI, 1999). Dans la dernière colonne figurent les niveaux de référence européens proposés en 1998 (Commission européennes, 1996).

Values of entrance surface dose measured by TLD in anthropomorphic RANDO phantom, for 10 examinations, in 3 hospital radiology departments in Paris (OPRI, 1999). The last column indicates european DRLs.

\begin{tabular}{|c|c|c|c|c|}
\hline 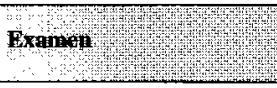 & Woptort & Hopital B & Hoptorto & Hapos \\
\hline Bassin (face) & 6,9 & 10,2 & 5,9 & 10 \\
\hline Abdomen sans préparakien & 4,9 & 10,9 & 2,4 & 10 \\
\hline Poumon (face) & 0,2 & 0.3 & 0.5 & 0.3 \\
\hline Cervical (face) & 1,5 & 4,2 & 0,5 & - \\
\hline Cervical (profil) & 3,2 & 5,8 & 0,5 & - \\
\hline Cervical $3 / 4$ & & 3 & 0,6 & - \\
\hline Rein & 10.7 & 8,5 & 3,9 & - \\
\hline L5-S1 $\quad \cdots$ & 6.1 & 12,7 & 6,6 & - \\
\hline 1.ombaire (face) & 5,7 & 8.4 & 3.8 & 10 \\
\hline Lombaire (protili) & 7,7 & 22,4 & 10 & 30 \\
\hline
\end{tabular}

professeurs A. Bonnin (AP/HP) et J.F. Lacronique (OPRI), (OPRI, 1999). Cette étude réalisée en collaboration avec les personnels de 3 services de radiologie de l'assistance publique des hôpitaux de Paris et du service de santé des armées a concerné 10 examens de radiologie conventionnelle, 20 patients par examen et a fourni 600 mesures exploitables. Son objectif n'était pas de déterminer des NRD par la méthode du $75^{\mathrm{C}}$ percentile, mais de comparer les doses moyennes obtenues dans chaque service, par type d'examen, aux valeurs de référence européennes. Les résultats publiés dans le rapport de la mission (OPRI, 1999) montrent que ces doses moyennes sont soit inférieures, soit du même ordre de grandeur que les niveaux européens. En complément, des mesures sur un fantôme anthropomorphe standard (Tab. III) ont été effectuées sur les mêmes installations pour les mêmes examens. Les résultats confirment ceux obtenus in vivo et montrent que la radiologie française ne se singularise pas en Europe du point de vue des doses délivrées aux patients.

Les niveaux de référence européens constituent donc un point de départ réaliste et efficace pour la pratique radiologique française. Ils ont été adoptés, et leur champ d'application élargi par le rapport OPRI-SFR (OPRI, 2001) : « Procédures radiologiques : critères de qualité et optimisation des doses " . 
La deuxième étape s'est ouverte avec la campagne nationale de mesures de doses en radiologie qui s'est mise en place, associant l'IRSN, la SFR, la Société française de physique médicale (SFPM) et des représentants des techniciens en électroradiologie médicale (AFPPE). Son objectif est d'associer des niveaux de doses aux procédures standardisées élaborées préalablement, et d'en déduire des valeurs de NRD propres à la France pour un certain nombre d'examens. Des protocoles de mesures dosimétriques ont été proposés pour 8 examens standard (4 en radiologie conventionnelle et 4 en scanographie) parmi les plus fréquents. Une trentaine de services de radiologie se sont portés volontaires pour appliquer ces protocoles, avec la collaboration de physiciens médicaux, sur des séries de 20 patients par examen. Il s'agit d'une phase de faisabilité, prévue entre avril 2001 et décembre 2002 (Naudy et al., 2002), qui devrait ouvrir la voie à une campagne de mesures à plus large échelle. La fourniture des dosimètres, la centralisation et le traitement des données sont assurés par le laboratoire de dosimétrie de l'IRSN (Service de surveillance de l'exposition médicale et professionnelle).

\section{Utilisation et évolution des niveaux de référence diagnostiques}

Utiliser les NRD c'est faire en sorte que dans tous les services de radiologie, sur chaque installation, une évaluation périodique des doses délivrées aux patients, pour des procédures courantes (sur des fantômes standard ou des groupes de patients types) soit réalisée. La dose moyenne, calculée par type d'examen, pour un nombre significatif de patients (20 au minimum) est comparée à la valeur du niveau de référence. Si les résultats montrent un dépassement régulier des NRD, une révision des procédures et un contrôle des installations s'imposent. Une action correctrice doit être envisagée si rien ne justifie le dépassement.

Rappelons cependant que le respect des niveaux de référence n'est pas le seul critère de bonne pratique. D'une part le principe de justification des actes doit au préalable être respecté, d'autre part, le non-dépassement des niveaux de référence ne dispense pas de poursuivre la démarche d'optimisation des doses, en gardant comme objectif permanent, indissociable de la dosimétrie, la qualité diagnostique des images.

\section{Conclusion}

Jusqu'à présent, les services d'imagerie français ayant réalisé des études dosimétriques sont peu nombreux. La mesure et l'analyse des doses délivrées (qui demande des moyens matériels et humains et une formation spécifique) ne font pas encore partie de la routine. La transposition effective de la directive 97/43 Euratom devrait changer progressivement cet état de fait, désormais en contradiction avec 
la réglementation. La mise en cuvre de la radioprotection des patients, et donc l'utilisation de niveaux de référence diagnostiques, sont devenus obligatoires. Un premier pas important a été fait avec la publication du rapport OPRI/SFR «Les procédures radiologiques : critères de qualité et optimisation des doses » (OPRI, 2001), qui propose une standardisation des examens, des recommandations pour réduire les doses et l'adoption des NRD européens comme point de départ pour une évaluation générale des pratiques. L'étude dosimétrique en cours constitue un second pas démontrant la faisabilité et l'intérêt d'une grande enquête nationale sur les doses délivrées en radiologie. Au-delà de l'engagement volontaire de quelques dizaines de centres, une telle enquête fournirait une base de données permettant de connaître le niveau réel de l'irradiation médicale en France et de faire évoluer les niveaux de référence en fonction de l'amélioration des pratiques, des techniques et des équipements.

\section{RÉFÉRENCES}

Commission européenne (1996) European guidelines on quality criteria foe diagnostic radiographic images. EUR 16260. Publications des communautés européennes, Luxembourg.

Commission européenne (1997) Quality criteria for computed tomography. EUR 16262. Publications des communautés européennes, Luxembourg.

Commission européenne (1999) Conseils sur la mise en œuvre de niveaux de référence diagnostiques pour les expositions médicales, Radioprotection 109, Publications des communautés européennes, Luxembourg.

Conseil des communautés européennes (1996) Directive 96/29/Euratom du 13 mai 1996 fixant les normes de base relatives à la protection sanitaire de la population et des travailleurs contre le danger des rayonnements ionisants, J.O. des communautés européennes n ${ }^{\circ} \mathrm{L} 159$.

Conseil des communautés européennes (1997) Directive 97/43/Euratom du 30 juin 1997 relative à la protection sanitaire des personnes contre les dangers des rayonnements ionisants lors des expositions à des fins médicales, J.O. des communautés européennes $n^{\circ} \mathrm{L} 180$.

Cordoliani Y.-S., Boyer B., Le Marec E., Jouan E., Helie O., Beauvais H. (2002) Vade-mecum du scanner hélicoïdal : estimation des doses, choix des paramètres, J. Radiol. 83(6), 685-692.

ICRP Publication 60 (1991) 1990 Recommendations of the International Commission on Radiological Protection, Ann. ICRP 21(1-3).

ICRP Publication 73 (1996) Radiological protection and safety in medicine, Ann. ICRP 26(2).

ICRP (2001) Supporting guidance, Diagnostic reference levels in medical imaging, review and additionnal advice, Ann. ICRP 30(2).

JO (2001a) Ordonnance 2001-270 du 28/03/01 relative à la transposition de directives communautaires dans le domaine de la protection contre les rayonnements ionisants.

JO (2001b) Décret 2001-1154 du 05/12/01 relatif à l'obligation de maintenance et au contrôle de qualité des dispositifs médicaux prévus à l'article L.5212-1 du code de la santé publique.

JO (2002) Décrets 2002-254 et 2002-255 du 22/02/02 relatif à l'Institut de radioprotection et de sûreté nucléaire et créant une direction générale de la sûreté nucléaire et de la radioprotection.

JO (2003) Décret 2003-270 du 24/03/03 relatif à la protection des personnes exposées à des rayonnements ionisants à des fins médicales et médico-légales. 
Maccia C., Benedittini M., Lefaure C. (1988) Doses to patients from diagnostic radiology in France, Health Phys. 54(4), 397-408.

Nagel H.D. et al. (2000) Radiation Exposure in Computed Tomography, European Coordination Committee of the Radiological and Electromedical Industries, Hamburg, German.

Naudy S., Valero M., Beauvais-March H., Hocine N. (2002) Campagne nationale de dosimétric en radiologie : résultats préliminaires, $41^{\text {es }}$ Journées scientifiques de la Société française de physique médicale, Grenoble, juin 2002, Publication SFPM.

NRPB (1999) Guidelines on patient dose to promote the optimisation of protection for diagnostic medical exposures, Doc. $N R P B 10, \mathrm{UK}$.

OPRI (1999) Rapport de mission des Professeurs A. Bonnin et J.-F. Lacronique sur l'irradiation médicale des patients, Publication OPRI.

OPRI (2001) Mission sur les procédures et les niveaux de référence des examens médicaux utilisant les rayonnements ionisants. Les procédures radiologiques : critères de qualité ct optimisation des doses.

SFPH (1988) Société française des physiciens d’hôpital, Évaluation des doses délivrées au cours d'examens radiologiques, Radioprotection $\mathbf{2 3}$. 